\title{
Outer divertor of ASDEX Upgrade in low-density L-mode discharges in forward and reversed magnetic field \\ Part I: Comparison between measured plasma conditions and SOLPS5.0 code calculations
}

\author{
L. Aho-Mantila ${ }^{1}$, M. Wischmeier ${ }^{2}$, H.W. Müller ${ }^{2}$, \\ S. Potzel $^{2}$, D.P. Coster ${ }^{2}$, X. Bonnin ${ }^{3}$, G.D. Conway ${ }^{2}$, \\ ASDEX Upgrade Team \\ 1 VTT Technical Research Centre of Finland, P.O.Box 1000, FI-02044 VTT, \\ Finland \\ 2 Max-Planck-Institut für Plasmaphysik, D-85748 Garching, Germany \\ ${ }^{3}$ LSPM - CNRS, Université Paris 13, F-93430 Villetaneuse, France \\ E-mail: leena.aho-mantila@vtt.fi
}

\begin{abstract}
The scrape-off layer and divertor plasma conditions have been carefully analysed in dedicated ASDEX Upgrade experiments consisting of lowdensity L-mode discharges, with both forward and reversed toroidal magnetic field and plasma current. In forward field, the outer divertor plasma is in a lowrecycling regime with peak target temperature above $25 \mathrm{eV}$. In reversed field with similar main plasma parameters, the target temperature is below $15 \mathrm{eV}$ and the density is 5 times as high as in forward field, indicating a higher recycling regime in the outer divertor. The SOLPS5.0 code package is used to model these divertor plasmas. Specifically, it is tested whether a combination of input assumptions exists that enables matching the code solution to all outer divertor diagnostic measurements, and whether these assumptions are compatible with constraints imposed by measurements outside the outer divertor. In forward field, a good level of agreement with multiple outer target measurements is found with assumptions that simultaneously match the measured density and temperature profiles at the outer midplane, where the uncertainty in radial position of the separatrix is $\pm 0.5 \mathrm{~cm}$. Similar approaches made previously for higher recycling regimes have not led to such a good consistency between all modelled and measured outer divertor parameters. In reversed field with higher recycling in the outer divertor, a solution consistent with the outer target Langmuir probe measurements cannot be obtained, at least not without significantly compromising the match to the upstream profile measurements. Significant mismatches are observed also between the modelled and measured upstream Mach number in forward field. These discrepancies question the global validity of the plasma solutions, and their origin is not yet clear. In Part II, the analysis of outer divertor conditions is complemented by local impurity migration studies, using the divertor plasma solutions presented in this paper.
\end{abstract}

Submitted to: Nuclear Fusion 


\section{Introduction}

2D multifluid edge code packages, like SOLPS [1], EDGE2D [2] and UEDGE [3], are often used to model the scrape-off layer (SOL) and divertor plasmas in both presentday tokamaks and future devices. When interpreting experiments, the models can be used to obtain a detailed $2 \mathrm{D}$ distribution of plasma parameters in between the measurement points, located typically at the targets and at the outer midplane, and to perform parametric studies of the SOL and divertor conditions. In addition, modelling allows making a valuable consistency check for the various diagnostic data, which helps to improve confidence in measurements. The fluid codes are also used for predictive studies, which explore the divertor operation [4] or provide background plasma models for more detailed impurity-following or plasma-wall interaction simulations [5] in future devices.

The major concern with respect to $2 \mathrm{D}$ edge fluid modelling is the observed inability of the codes to reproduce several key observations made in present experiments. Severe discrepancies have been observed, for example, when simulating divertor detachment [6] or SOL plasmas with medium collisionality [7]. Despite efforts made to include new physics, by introducing various mock-up models [8] or e.g. new formulation of anomalous transport [9], the underlying reasons for these discrepancies have not been resolved up to date, limiting the predictive capability of the codes. To test whether these discrepancies are specific to the recycling regime, we have focused in this paper on dedicated low-density L-mode discharges, in which the difficult highrecycling and detached divertor regimes are avoided, at least in the typically more attached outer divertor plasma.

The experiments were performed in the ASDEX Upgrade (AUG) tokamak, which is close to a geometrically scaled-down version of ITER [10]. An ITER-like plasma geometry and fully $\mathrm{W}$-coated plasma-facing components were in use in these experiments. To model the experiments, we have used the SOLPS5.0 code package, with the plasma fluid code B2.5 iteratively coupled to the Monte Carlo neutrals code Eirene. This code package has the benefit that calculation of electric currents and drifts $(\mathbf{E} \times \mathbf{B}$ and diamagnetic) can be activated in the simulations in the presence of intrinsic impurities (residual $\mathrm{C}$ in $\mathrm{AUG}$ ). It is shown that when the toroidal magnetic field, $B_{\mathrm{T}}$, and plasma current, $I_{\mathrm{p}}$, are reversed in the experiments, the outer divertor conditions change significantly. Therefore, all the simulations presented in this paper are performed with the drift terms fully activated.

The main focus of this paper is on the outer divertor plasma. Extensive parameter scans have been performed to investigate whether a SOLPS5.0 solution exists that matches the multiple measurements in the outer divertor and, thus, whether the diagnostic data are consistent. As experimental upstream boundary conditions the measured heating power to the scrape-off layer and plasma density and temperature profiles at the outer midplane are used. Since these measurements are subject to uncertainties, a range of boundary conditions for the divertor modelling has been investigated. Additional assumptions, such as recycling and sputtering at the target plates, have been validated against experimental signals where possible. For completeness, comparisons of the solutions to additional upstream measurements, such as the parallel Mach number, and to those in the inner divertor are presented. Part II studies local impurity migration in the outer divertor in the same experiments, for which reason carefully validated outer divertor plasma models are sought for in the present paper. 
Table 1. Summary of discharges analysed in this paper. Discharges in bold include radial and vertical shifts of the plasma equilibrium for diagnostic measurements.

\begin{tabular}{lcccccc}
\hline discharge numbers & year & $\begin{array}{c}B_{\mathrm{T}} \\
(\mathrm{T})\end{array}$ & $\begin{array}{c}I_{\mathrm{p}} \\
(\mathrm{MA})\end{array}$ & $\begin{array}{c}\bar{n}_{\mathrm{e}} \\
\left(\times 10^{19} \mathrm{~m}^{-3}\right)\end{array}$ & $\begin{array}{c}P_{\mathrm{ECRH}} \\
(\mathrm{MW})\end{array}$ & $\begin{array}{c}P_{\mathrm{Ohmic}} \\
(\mathrm{MW})\end{array}$ \\
\hline $\begin{array}{l}\text { Forward field } \\
\text { \#22469, \#22573-85* }\end{array}$ & 2007 & -2.5 & 0.8 & 3.2 & 0.60 & 0.35 \\
$\begin{array}{l}\text { \#24178, \#24183, \#24187-9 } \\
\text { \#24908, \#24911 }\end{array}$ & 2008 & -2.5 & 0.8 & 3.2 & 0.65 & 0.30 \\
$\quad 2009$ & -2.5 & 0.8 & 3.0 & 0.60 & 0.35 \\
$\begin{array}{l}\text { Reversed field } \\
\text { \#25881, \#25883-90 }\end{array}$ & 2009 & 2.5 & -0.8 & 3.4 & 0.90 & 0.30 \\
\hline
\end{tabular}

${ }^{*}$ Excluding \#22576,-82

The paper is organized as follows. Section 2 describes the experiments and the setup used in the simulations. In Sections 3 and 4, the modelled divertor plasmas are compared to experimental data in forward and reversed field, respectively. Section 5 completes the benchmarking by showing comparisons between measured and modelled parallel ion flows and radial electric field at the outer midplane. Conclusions are presented in section 6 .

\section{Description of the experiments and setup for the simulations according to the upstream measurements}

This paper focuses on a series of low-density L-mode discharges performed in ASDEX Upgrade in 2007-2009. The discharges were all performed in a lower-single-null topology with deuterium main plasma species and ECRH heating. Most of the discharges were connected to ${ }^{13} \mathrm{C}$ injection experiments, which were carried out at the end of the experimental campaigns, just before vessel openings (excluding \#24908 and \#24911). In these experiments, ${ }^{13} \mathrm{CH}_{4}$ was injected at a trace level from the outer divertor, and in 2007 also from the outer plasma midplane. The impurity injection was not observed to influence the diagnostic measurements.

To model the experiments, 2-dimensional calculation grids covering the main SOL, divertor plasma, and private flux region, PFR, were created according to the equilibrium reconstruction by CLISTE [11], using the grid generator CARRE [12]. In order to perform the relevant parameter scans, the resolution of the grids was limited to 48 grid points in the poloidal direction and 18 grid points in the radial direction, as shown in figure 1 for the reversed field configuration. For numerical stability, the very narrow inner PFR was extended in the forward field simulations by reducing the width of the leftmost divertor dome segment. This extended distance between the inner strike point and the dome is expected to have a negligible effect on the plasma solution outside the inner PFR.

Anomalous transport was included in the SOLPS5.0 simulations by specifying a priori radially varying coefficients for particle diffusion, $D_{\perp}$, same for all species, and electron and ion heat conduction, $\chi_{\perp \mathrm{e}}$ and $\chi_{\perp \mathrm{i}}$, respectively. Besides ballooning along the flux surfaces $\left(\propto B^{-1}\right)$, no poloidal variation of the transport coefficients was attempted within the present work. These assumptions, together with the estimated 


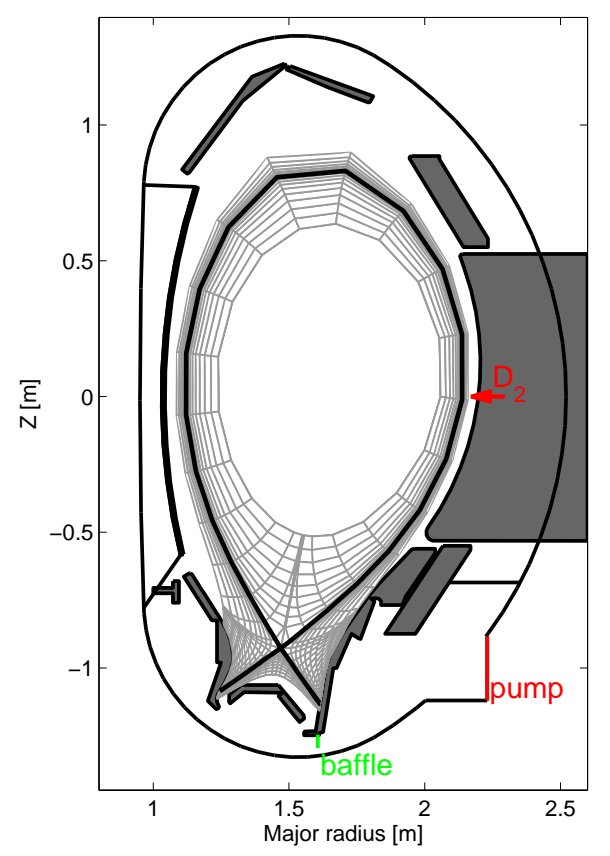

Figure 1. SOLPS grid and vessel segments used for modelling the reversed field discharges. The positions of the gas puff and pump are drawn in red. A baffle was introduced in the simulations to match the ratio of neutral conductivities in the different subdivertor regions.

particle and power input to the scrape-off layer, largely determine the particle and power fluxes in the divertor and, hence, the recycling regime. For benchmarking these global assumptions, we begin the analysis by considering the measurements made outside the divertor.

The power entering the simulation regime from the core plasma, $P_{\text {in }}$, can be estimated by subtracting the measured core radiation from the total heating power in the experiments. In earlier publications of the 2007 forward field experiment [13,14], small temperature gradients were modelled along the field lines on the low-field side, LFS, leading to significantly higher outer target temperatures compared to the measurements. Therefore, we have carefully reviewed the assumptions of $P_{\text {in }}$ in this paper, using additional experimental data from 2008. Figure 2 shows the typical time traces observed during the first two experiments in forward field and the last one with reversed field. The accuracy of the absolute calorimetric calibration of the ECRH and the assumptions on transmission losses for the torus window and launcher [15] allow for an error of $5 \%$ to $10 \%$ in the time traces. Identical ECRH sources were used in the two forward field discharges, apart from the third source which was only used in the latter. According to the time traces, the third source has $P_{\mathrm{ECRH}}=0.70 \mathrm{MW}$, which is the maximum available power for that source and can be considered as a realistic measurement. However, in contrast to the measured variation of $P_{\mathrm{ECRH}}, T_{\mathrm{e}}$ in $\# 24183$ is observed to raise at two distinct steps, indicating an increase in the deposited ECRH power for the second and third source. Therefore, we conclude that the time traces 

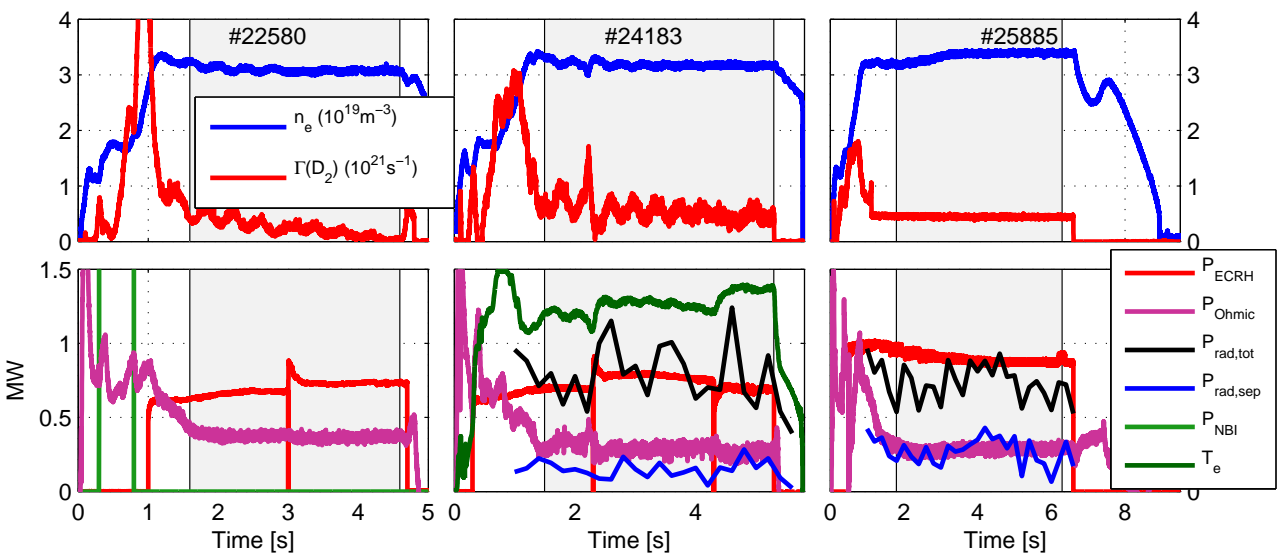

Figure 2. Typical time traces in the first two forward field experiments (left, middle) and the reversed field experiment (right). Upper row: line-averaged density and midplane $\mathrm{D}_{2}$ gas puff. Lower row: heating and radiated powers. The time intervals when ${ }^{13} \mathrm{CH}_{4}$ was injected are highlighted, to support the analysis that will be presented in Part II. The lower middle plot shows the $T_{\mathrm{e}}$ evolution (a.u.), measured at $\rho \sim 0.8$ (qualitatively similar $T_{\mathrm{e}}$ time traces were obtained throughout the plasma in \#24183). The NBI power in the beginning of \#22580 was $2.5 \mathrm{MW}$.
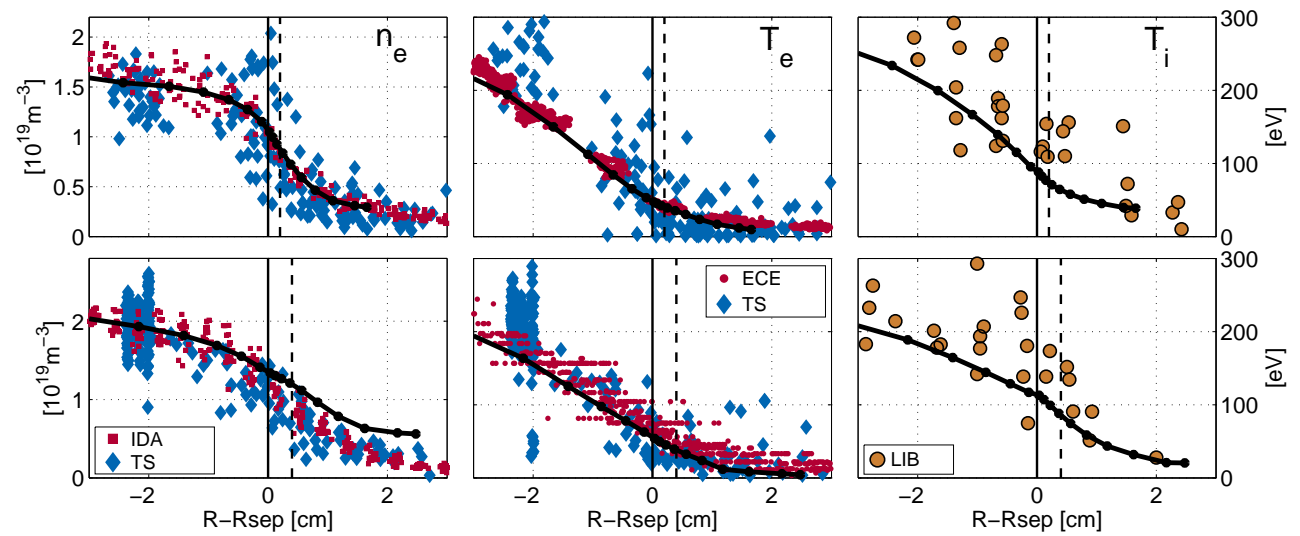

Figure 3. Modelled and measured profiles of $T_{\mathrm{e}}, T_{\mathrm{i}}$ and $n_{\mathrm{e}}$ at the outer midplane in forward (upper row) and reversed field (lower row). The data is collected from the 2007 discharges in forward field and the 2009 discharges in reversed field, see table 1. The TS data shown for forward field are from discharges \#24908 and \#24911. The black lines represent the SOLPS5.0 solutions. The vertical lines indicate the original (dashed line) and shifted (solid line) separatrix positions. 


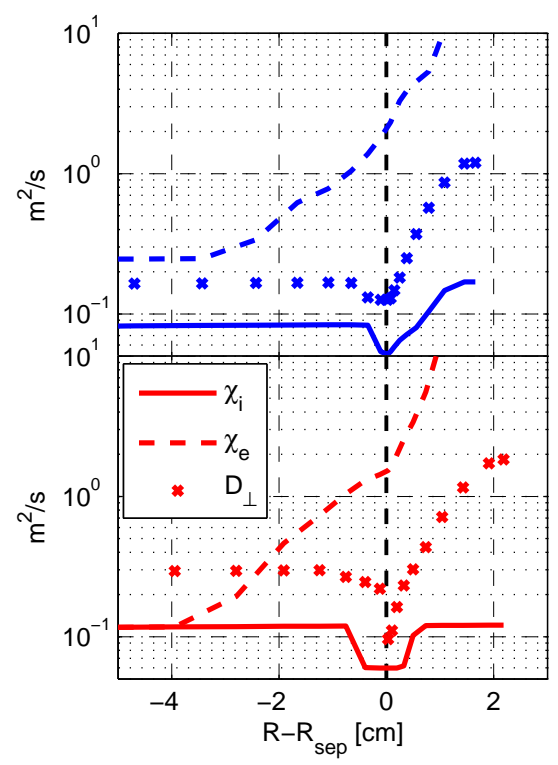

Figure 4. Coefficients used in the simulations for forward (upper) and reversed field (lower) for radial particle diffusion, $D_{\perp}$, and radial ion and electron heat conductivities, $\chi_{\perp \mathrm{i}}$ and $\chi_{\perp \mathrm{e}}$, respectively.

must give an overestimated power for the first two sources. We estimate that the power from the first two sources is $10 \%$ lower than that shown in figure 2 . The maximum available $P_{\mathrm{ECRH}}=0.90 \mathrm{MW}$ in 2009 is estimated for the reversed field experiment.

Figure 2 also shows the total radiated power, $P_{\text {rad,tot, }}$, and the power radiated on flux surfaces with $\rho<0.95, P_{\text {rad,sep }}$, derived from a convolution of bolometer measurements in the last two experiments. For the first experiment, radiation similar to \#24183 is assumed. Accordingly, we estimate $P_{\text {in }} \sim 0.70 \mathrm{MW}$ in forward field and $P_{\text {in }} \sim 1.0 \mathrm{MW}$ in reversed field. The first estimate is lower than the ones used in $[13,14]$, improving the agreement between modelled and measured edge $T_{\mathrm{e}}$, shown later.

The upstream particle input is determined by the midplane $\mathrm{D}_{2}$ gas puff, which was included in the simulations together with the subdivertor pump, shown in figure 1. In the forward field experiments, the gas puff was operated in a feedback mode for $\bar{n}_{\mathrm{e}}$, which resulted in a small oscillation of $\bar{n}_{\mathrm{e}}$. In reversed field, the gas puff was changed to a feedforward mode, and an increase in $\bar{n}_{\mathrm{e}}$ from $3.2 \times 10^{19} \mathrm{~m}^{-3}$ to $3.4 \times 10^{19} \mathrm{~m}^{-3}$ during the first 2 seconds of the flattop was observed. The measured $\mathrm{D}_{2}$ pumping rate below the divertor was lower than the puffing rate at the midplane, and a steady increase in sub-divertor neutral fluxes was measured during the reversed field discharges. These observations indicate that the walls were not fully saturated by neutrals but acted as an additional 'pump' by absorbing the neutrals, thereby reducing the recycling at the walls and thus likely explaining the slow ramp-up of $\bar{n}_{\mathrm{e}}$. Instead of matching the measured puffing and pumping rates, which would require a detailed match to the experimental conditions in both divertor legs, the simulated puff was operated in a feedback mode for the upstream separatrix density, $n_{\text {sep }}$. The possible values of $n_{\text {sep }}$ were determined from the measured upstream profiles, as follows. 
Figure 3 shows the modelled and measured profiles for $n_{\mathrm{e}}, T_{\mathrm{i}}$ and $T_{\mathrm{e}}$ at the outer plasma midplane in forward and reversed field. The experimental data are from the Thomson scattering system, TS, the ECE diagnostics and the lithium beam, LIB. The figure includes a correction of the density measurements by TS in reversed field by a factor of 0.7 , to compensate a calibration error in the system at the time of these discharges. The IDA (integrated data analysis) profiles combine the measurements by LIB and laser interferometry [16].

It is important to note, that the relative radial positions of the profiles measured by different diagnostics are subject to uncertainties. The most reliable way of deducing the relative positions of the $n_{\mathrm{e}}$ and $T_{\mathrm{e}}$ profiles is by using good quality TS measurements for both quantities. However, the profiles often have a large scatter of data points, which makes fitting of the profiles particularly challenging for L-mode plasmas without a clear pedestal. An example of reliable use of Thomson scattering data to deduce the relative positioning of the $n_{\mathrm{e}}$ and $T_{\mathrm{e}}$ profiles in $\mathrm{H}$-mode plasmas can be found in [7]. In the present paper, the ECE profile in forward field has been shifted by $3 \mathrm{~mm}$ towards the core with respect to the Li-beam measurements, which is the average misalignment between the two profiles, deduced by analysing a wide range of discharges with good-quality TS measurements [17]. The sensitivity of the solution with respect to this relative shift is discussed in Section 3. The TS data are shifted to match the Li-beam density measurements, after which they coincide also with the ECE profile. In reversed field, no misalignment between ECE and Li-beam is assumed to achieve agreement with the profiles from TS.

The absolute position of the separatrix can be further shifted within the experimental uncertainty range $( \pm 5 \mathrm{~mm})$, which means that the measurements allow for a range of possible values of $n_{\mathrm{sep}}$. Varying $n_{\mathrm{sep}}$ within this range changes the conditions in the outer divertor. In the present work, the absolute position of the separatrix was varied in order to find a value of $n_{\text {sep }}$ that yields the best agreement between modelled and measured outer divertor conditions. It is worth noting, that a good agreement with all outer divertor measurements is rarely achieved by such variations. In both field directions, the best match to outer divertor conditions was achieved with a larger $n_{\text {sep }}$ than indicated by the equilibrium calculations and, therefore, the separatrix positions were shifted accordingly, see figure 3. In forward field, $n_{\text {sep }}=1.1 \times 10^{19} \mathrm{~m}^{-3}$ was specified ( $2 \mathrm{~mm}$ shift), and the measured upstream $n_{\mathrm{e}}$ profile is reproduced well when assuming a low $D_{\perp}$ that increases rapidly in the SOL, see figure 4 . In reversed field, similar transport assumptions give a flatter simulated $n_{\mathrm{e}}$ profile. When $n_{\mathrm{sep}}=1.4 \times 10^{19} \mathrm{~m}^{-3}$ is specified (4 mm shift), the simulations give slightly higher densities in the SOL than what is measured. This small discrepancy is deliberately left in the upstream profile, in order to bring the outer target density profiles closer to measured values, discussed later. The measured $T_{\mathrm{e}}$ profiles are reproduced in both experiments by assuming anomalously large electron heat conduction in the SOL. The heat conduction of ions is assumed to be of the neoclassical order of magnitude.

\section{Divertor conditions in forward field}

Further to the set-up described up to now, the conditions in the divertor can be influenced by assumptions on plasma-wall interaction, such as recycling and impurity sources. Despite the full-tungsten wall, carbon deposits exist in the divertor of AUG. To match the carbon impurity radiation, the simulations were carried out with a 

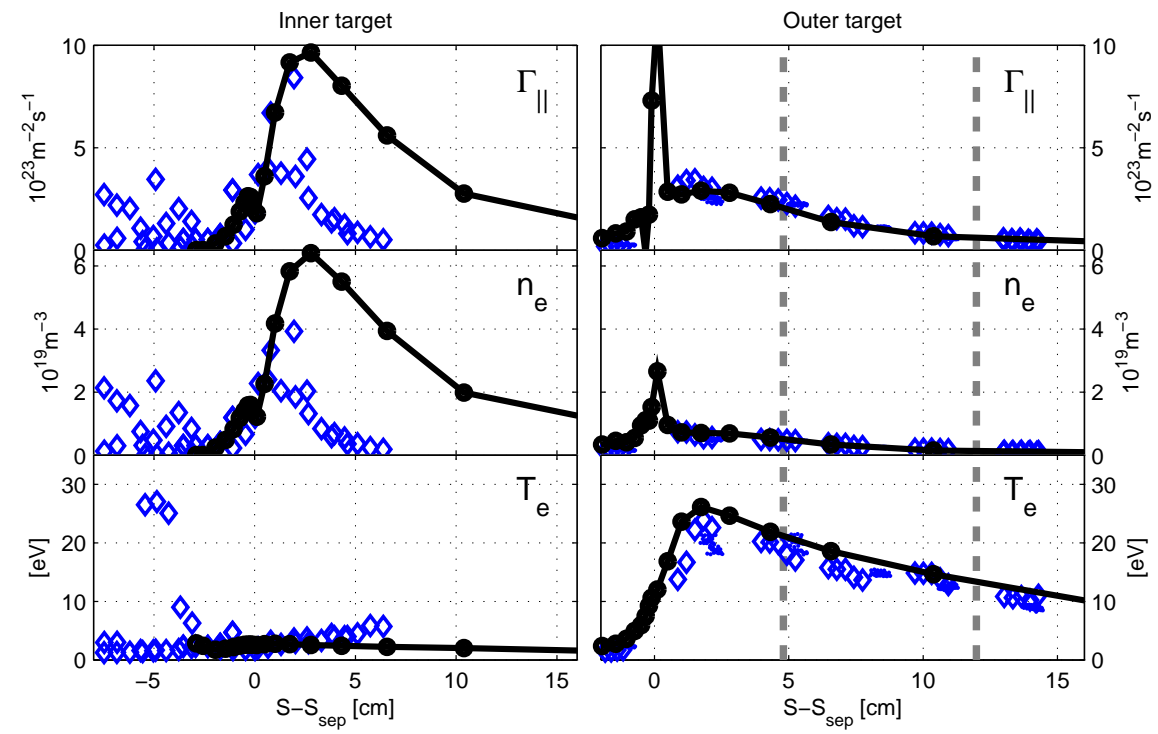

Figure 5. Target $\Gamma_{\|}, T_{\mathrm{e}}$ and $n_{\mathrm{e}}$ modelled with SOLPS5.0 (black lines) and measured by the flush-mounted Langmuir probes in AUG (blue diamonds; outer target: \#22469, inner target: \#24911) in forward field. To ease the comparison, the data for the two targets are shown on the same scale. The dashed grey lines indicate the positions of the two ${ }^{13} \mathrm{CH}_{4}$ injection valves.

full-carbon wall that undergoes physical and chemical erosion. The yield of chemical erosion, $Y_{\text {chem }}$, was varied to match the measured signals.

The primary measured data from the divertor plasma come from the triple and single Langmuir probes, LP, which in AUG are flush-mounted with the divertor tiles. At the divertor targets, the plasma model in SOLPS5.0 extends until the entrance to the magnetic presheath, MPS. At this boundary, the parallel velocity of each ion species is constrained by the modified Bohm-Chodura boundary condition [18]:

$$
v_{\|} \geq c_{\mathrm{s}}-\frac{B_{\mathrm{tor}}}{B_{\mathrm{pol}}} v_{\perp, E \times B}
$$

where $B_{\text {tor }} / B_{\text {pol }} \sim 30$ is the inverse of the magnetic field pitch, $v_{\perp, E \times B}$ is the poloidal $\mathbf{E} \times \mathbf{B}$ drift velocity and $c_{\mathrm{s}}$ is the ion sound speed, defined as:

$$
c_{\mathrm{s}}=\sqrt{\left(n_{\alpha} Z_{\alpha} T_{\mathrm{e}} / n_{\mathrm{e}}+\gamma T_{\mathrm{i}}\right) m_{\alpha}^{-1}} .
$$

Here, $\gamma$ is the ratio of specific heats and $Z_{\alpha}, n_{\alpha}$ and $m_{\alpha}$ are the charge number, density and mass of the ion species, respectively. The probes measure $T_{\mathrm{e}}$ and the ion saturation current, $I_{\text {sat }}$, from which the parallel ion flux density, $\Gamma_{\|}$, and $n_{\mathrm{e}}$ can be derived. For comparison with the modelling, we note the following deviations in boundary assumptions: (i) The LP density is derived according to $\Gamma_{\|}=n_{\mathrm{e}} c_{\mathrm{s}}$ and, therefore, does not include the drift effects in (1) and assumes sonic flow without impurities; (ii) Standard AUG data evaluation assumes $Z=1, T_{\mathrm{i}}=T_{\mathrm{e}}$ and $\gamma=3$ (1D collisionless adiabatic flow) [19], whereas the simulations assume $\gamma=5 / 3$ (collisional adiabatic flow) in (2). For consistency, the LP $n_{\mathrm{e}}$ profiles presented in this paper are derived using $\gamma=5 / 3$. 

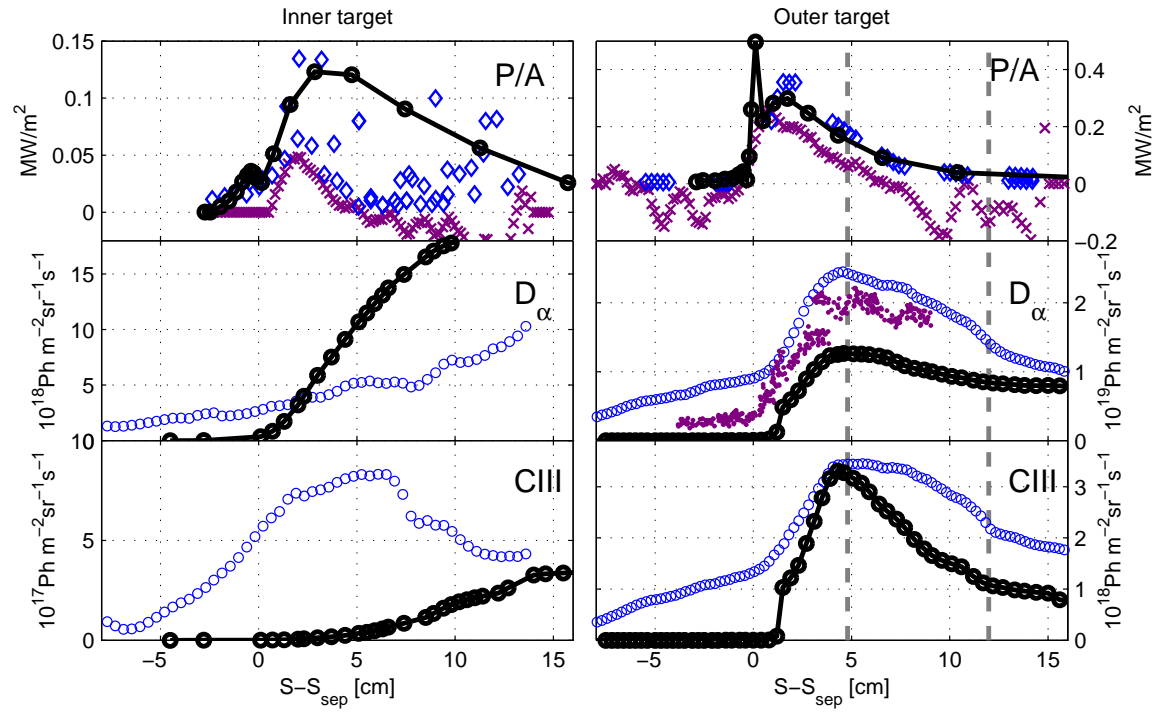

Figure 6. Modelled and measured power densities $(P / A)$ and line emission $\left(\mathrm{D}_{\alpha}\right.$, CIII at $\left.465 \mathrm{~nm}\right)$ in forward field. The crosses represent the data derived from the IR camera (\#25885), the diamonds correspond to the Langmuir probe data (outer target: \#22469, inner target: \#24911), and the circles give the spectroscopic measurements (\#22469). The magenta dots give the measured emission in \#24911. The SOLPS5.0 solutions are drawn with the solid black lines.

Figure 5 shows the comparison between simulated and measured $\Gamma_{\|}$(for $\mathrm{D}^{+}$only, the impurities have a negligible contribution), $T_{\mathrm{e}}$ and $n_{\mathrm{e}}$ at the two divertor targets in forward field, with the inner target data obtained from the 2009 discharges (in 2007-8 the inner probes were not measuring correctly because of too shallow magnetic field incidence angle). The code solution is matched to the measured low recycling outer target conditions, with a peak electron temperature $T_{\mathrm{e}, \mathrm{ot}}^{\mathrm{pk}}=25 \mathrm{eV}$. (The LP biasing voltage $-72 \mathrm{~V}$ may have been marginal around the strike point, but should have been sufficient close to the valve locations.) Particularly at the locations of ${ }^{13} \mathrm{CH}_{4}$ injection, both the absolute value and the gradients along the target are reproduced in the simulations for all three measured quantities. $T_{\mathrm{e}, \mathrm{ot}}^{\mathrm{pk}}$ is reduced compared to previous modelling efforts in $[13,14]$, which is largely a result of changes in assumptions for $P_{\text {in }}, n_{\text {sep }}$, and cross-field transport, with minor contribution from code development. The LP data do not cover the whole strike point and, thus, cannot be used to verify the modelled density peak at that location. At the inner target, both modelling and measurements indicate a high recycling regime with $T_{\mathrm{e}, \text { it }}^{\mathrm{pk}}<5 \mathrm{eV}$, with density peaking close to the separatrix. The peak values of $\Gamma_{\|}$and $n_{\mathrm{e}}$ are, however, a factor of 2 higher in the simulations compared to the LP measurements. Similar mismatches have been observed in the past when modelling high recycling to detached conditions, pointing towards a common, presently unidentified, origin of discrepancy [8].

The simulations were compared to the power fluxes calculated from surface temperature measurements by an infrared camera, IR. Figure 6 shows this comparison, including also the power flux density calculated from the probe data: $P / A=$ $\left(8 k_{\mathrm{B}} T_{\mathrm{e}}+E_{\mathrm{rec}}\right) \tilde{\Gamma}_{\|}[20]$, where $\tilde{\Gamma}_{\|}$is now the flux through the probe surface and $E_{\text {rec }}$ is 
the energy released in surface recombination. At the outer target, a good agreement between the measured and modelled $P / A$ is obtained, as the deviations between IR and LP measurements are within the measurement uncertainties. At the inner target, $T_{\mathrm{e}, \mathrm{it}}^{\mathrm{pk}}$ is so low that the contribution from $E_{\mathrm{rec}}$ is comparable to the heat flux. The overestimation of $P / A$ by a factor of 2 in the modelling appears to be a direct consequence from the overestimation of $\Gamma_{\|}$. The good agreement between the IR (from 2007) and LP (from 2009 at the inner target) power flux measurements supports the low $n_{\mathrm{e}, \mathrm{it}}^{\mathrm{pk}}$ from the LP measurements. The measured peak power fluxes are a factor of 4 lower at the inner target, compared to the outer target.

While the LP and IR measurements can only be used to validate the modelled conditions along the targets, comparison of the $2 \mathrm{D}$ divertor solution can be made with respect to spectroscopic measurements. In the 2007 forward field discharges (\#22469), the $\mathrm{D}_{\alpha}$ line and the CIII line at $465 \mathrm{~nm}$ were measured with the SPO diagnostic along poloidal arrays of lines-of-sight, LOS, in both divertor legs, see figure 3.1 in [21]. To estimate the fraction of light that originates from reflection at the tungsten tiles [21], we have compared the $\mathrm{D}_{\alpha}$ emission with measurements in \#24911, which were carried out viewing the non-reflecting gaps between the tungsten tiles. This comparison indicates that the reflection adds an approximately constant amount, of the order of the PFR emission, to the measured outer target emission in \#22469. Hence, the modelled outer target $\mathrm{D}_{\alpha}$ emission agrees with the measured emission within $50 \%$, matching closely the measured profile along the target. In order to agree with the measured CIII line, $\mathrm{Y}_{\text {chem }}=0 \%$ was assumed in the simulations. At the inner target, agreement with the measured $\mathrm{D}_{\alpha}$ emission is only within a factor of 3 , which is likely to be associated with the discrepancies in $n_{\mathrm{e}, \mathrm{it}}$. The CIII emission is underestimated even when testing assumptions of $Y_{\text {chem }}$ up to $2 \%$. The surface temperatures, $T_{\text {surf }}$, at the two targets vary within $300-350 \mathrm{~K}$, in which range $\mathrm{Y}_{\text {chem }}$ should be low and fairly insensitive to $T_{\text {surf }}[22]$. The match could, however, be sensitive to the uneven distribution of carbon re-deposits which, furthermore, may be hydrogen-rich and undergo an enhanced re-erosion [23].

Finally, we have assessed the influence of a possible misalignment between the upstream density and temperature profiles on the modelled outer divertor parameters. As discussed in Section 2, a radial shift of $3 \mathrm{~mm}$ was assumed between the Li-beam and ECE measurements. The effect of this shift on target $T_{\mathrm{e}}$ and $n_{\mathrm{e}}$ is estimated based on a modified two-point model [20], with an effective loss factor calculated using the SOLPS5.0 solution presented in this paper. First, we note that a reduction in upstream $T_{\mathrm{e}}$ would correspond to a reduction in $P_{\mathrm{in}}$ to values which are outside the uncertainty range of the measurements (recall that $P_{\text {in }}$ was assumed to be at the lower limit of this uncertainty range, Section 2). Therefore, only misalignments of the density profile are considered. Assuming a higher $n_{\mathrm{sep}}=1.25 \times 10^{19} \mathrm{~m}^{-3}$, which corresponds to assuming the maximum possible radial shift of $5 \mathrm{~mm}$ of the measured separatrix position (and no shift between ECE and Li-beam measurements), only a slightly cooler and denser outer divertor plasma is obtained according to the two-point model ( $T_{\mathrm{e}, \mathrm{ot}}^{\mathrm{pk}} \sim 19 \mathrm{eV}$, a factor of 0.73 colder and $n_{\mathrm{e}, \mathrm{ot}}^{\mathrm{pk}} \sim 1.1 \times 10^{19} \mathrm{~m}^{-3}$, a factor of 1.6 denser). We conclude that these changes are too small to significantly question the validity of the outer divertor solution presented here.

To summarize, a good agreement with all outer divertor measurements could be achieved in these forward field discharges when using assumptions and boundary conditions compatible with the measured upstream $n_{\mathrm{e}}$ and $T_{\mathrm{e}}$ profiles and power to the scrape-off layer, within their uncertainty ranges. This indicates that the 

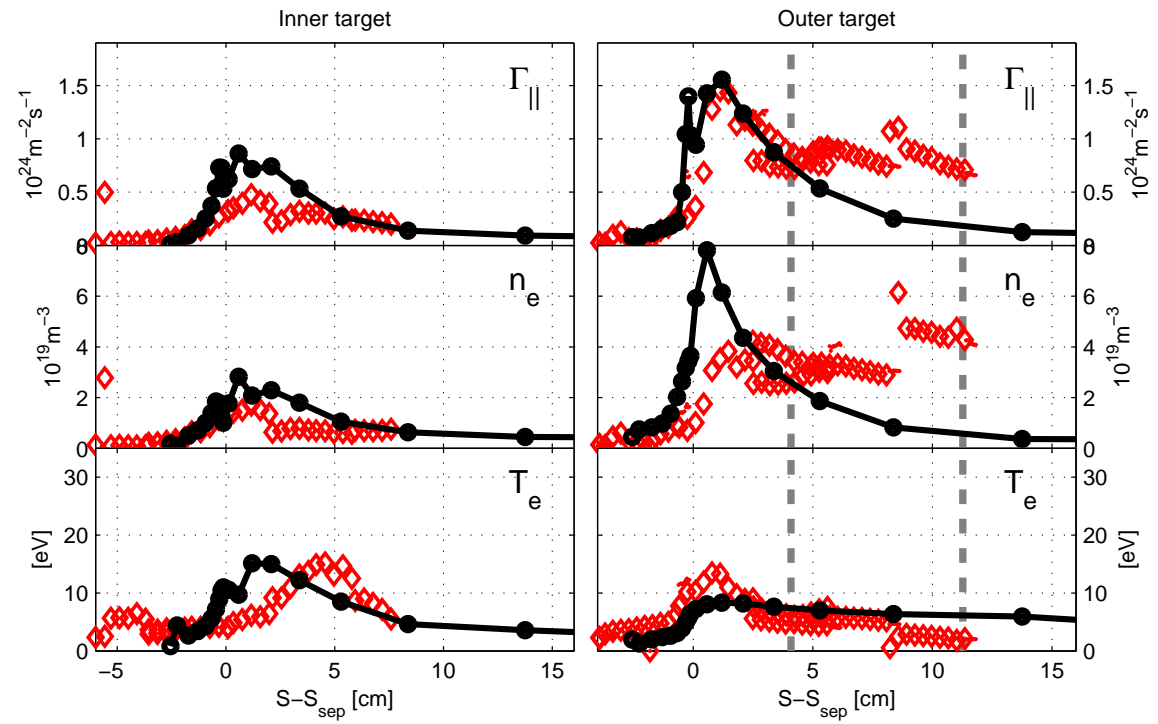

Figure 7. Target $\Gamma_{\|}, T_{\mathrm{e}}$ and $n_{\mathrm{e}}$ modelled with SOLPS5.0 (black, solid lines) and measured by the Langmuir probes (red diamonds, \#25881) in reversed field.

various divertor diagnostics yield consistent data in these low-recycling conditions. Simultaneously, typical discrepancies between the code and experiment are observed in the high-recycling inner divertor, with the modelled density exceeding the measured one by a factor of 2 . However, it is assumed that these discrepancies are not significant enough to question the validity of the outer divertor solution, in light of local impurity migration modelling.

\section{Divertor conditions in reversed field}

Despite the similarity in upstream measurements between the two field directions, large changes are observed in the divertor plasmas when $B_{\mathrm{T}}$ and $I_{\mathrm{p}}$ are reversed. Figure 7 shows the comparison between the simulations and the Langmuir probe measurements during the strike point scan in \#25881. At the outer target, the measured $n_{\mathrm{e}}$ and $\Gamma_{\|}$are a factor of 5 larger compared to forward field. The simultaneously high $T_{\mathrm{e}, \mathrm{ot}}^{\mathrm{pk}}$, above $10 \mathrm{eV}$, indicates that the plasma is in a mediumrecycling regime, i.e. it has not yet entered the cold and dense high-recycling regime. The probes measure a peak in $\Gamma_{\|}$and $T_{\mathrm{e}}$ close to the outer strike point but around the two ${ }^{13} \mathrm{CH}_{4}$ injection locations the profiles remain rather flat. The derived values of $n_{\mathrm{e}}$ are approximately constant along the outer target, apart from the upper valve location, where the probe measurements indicate an abrupt increase in $n_{\mathrm{e}}$. It is likely that this probe was not measuring correctly, raising an uncertainty of the plasma conditions in that location.

The simulations yield a satisfactory agreement with the measured particle loads on the outer target in reversed field. However, the modelled density profile is narrower compared to the measured one, with the peak $n_{\mathrm{e}, \mathrm{ot}}^{\mathrm{pk}}$ a factor of 2 larger compared to the experiment. A further increase in cross-field diffusion to flatten the target $n_{\mathrm{e}}$ profile would lead to intolerable discrepancies with the measured upstream $n_{\mathrm{e}}$ 


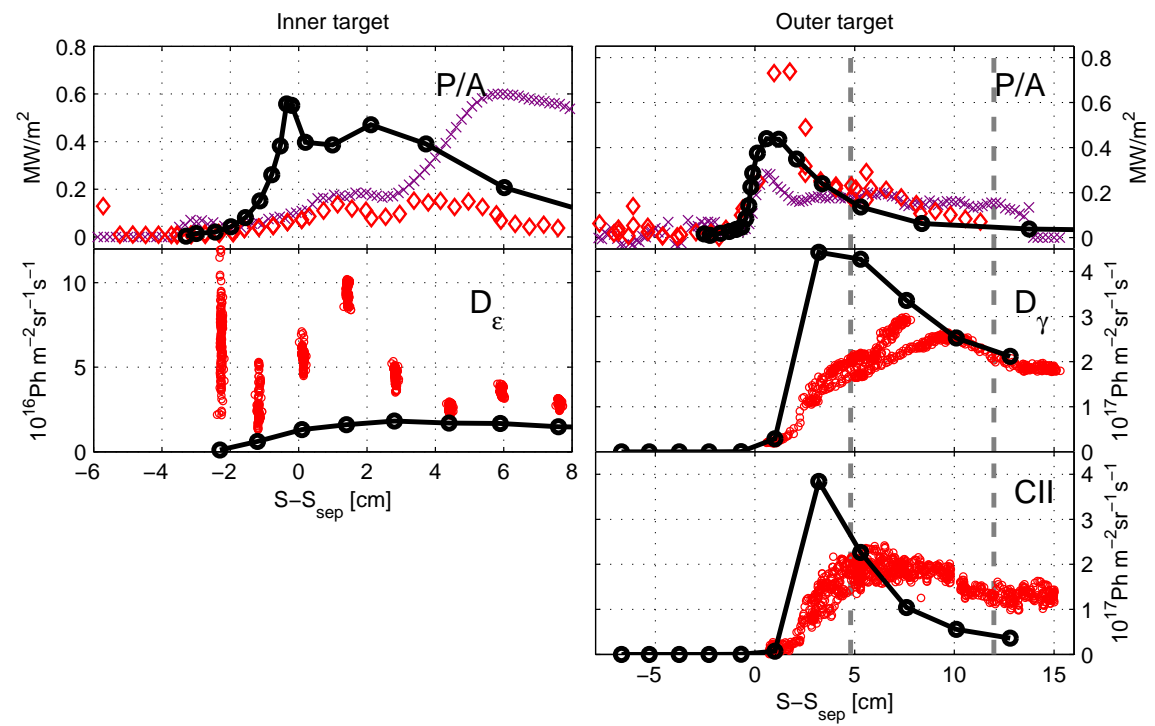

Figure 8. Power flux densities (P/A) and line emission $\left(D_{\epsilon}, D_{\gamma}, \mathrm{CII}\right.$ at $\left.427 \mathrm{~nm}\right)$ along the targets in Ex $x_{r e v}$. The crosses represent the data derived from IR camera, the diamonds correspond to the Langmuir probe data, and the circles give the spectroscopic measurements. The SOLPS solutions are drawn with the solid black lines.

profile, discussed in section 2. This suggests that in the experiment the transport towards the outer SOL occurs further downstream, at a poloidal location closer to the divertor target, where it leads to the flat density profile. This observation could not be reproduced in the present simulations, and further efforts are on-going to identify the relevant physical mechanisms. The solutions presented in figure 7 assume $98.5 \%$ recycling at all wall elements to mimic the wall pumping discussed in section 2 . With this assumption, a good agreement with the measured target $T_{\mathrm{e}}$ is obtained, but the modelled density peak at the strike point reduces the peak $T_{\mathrm{e}, \mathrm{ot}}^{\mathrm{pk}}$ below the measured value. Both measurements and modelling give similar values for the peak heat flux as in forward field, see figure 8, but the measured flat profiles of heat flux and emission $\left(D_{\gamma}\right.$ and CII at $\left.427 \mathrm{~nm}\right)$ are not reproduced in the simulations, similarly to the $n_{\mathrm{e}}$ and $\Gamma_{\|}$disagreements. Consequently, a reasonable agreement between the modelled and measured conditions is obtained only around the lower ${ }^{13} \mathrm{CH}_{4}$ injection location, whereas discrepancies are observed further outwards in the SOL.

At the inner target, a satisfactory agreement is obtained with the LP measurements of $\Gamma_{\|}$and $n_{\mathrm{e}}$. It should be noted that these measurements are unaffected by the field reversal in this low-density configuration. The simulations reproduce the measured density asymmetry in reversed field, which is a factor of 2 difference in density between the two targets. Nevertheless, a small deviation in the position of the peak temperature $T_{\mathrm{e}, \mathrm{it}}^{\mathrm{pk}}$ is observed. Measurements from the inner target show that, when $\bar{n}_{\mathrm{e}}$ increases from $3.2 \times 10^{19} \mathrm{~m}^{-3}$ to $3.4 \times 10^{19} \mathrm{~m}^{-3}$ during the discharge, recycling increases and $T_{\mathrm{e}}$ at the strike point drops from $20 \mathrm{eV}$ to $5 \mathrm{eV}$. More stationary conditions are observed at $\sim 5 \mathrm{~cm}$ distance from the separatrix, where the peak $T_{\mathrm{e}, \mathrm{it}}^{\mathrm{pk}} \sim 15 \mathrm{eV}$ is measured. In the simulations, the reason why the field reversal does not fully reverse the $T_{\mathrm{e}}$ asymmetry is found to be the radiation of carbon impurities 
close to the X-point, which results in power losses on the way to the inner strike point. Physical sputtering alone has a large impact on the solution but, additionally, $Y_{\text {chem }}=0.5 \%$ was specified at the inner target where typically thick re-deposited carbon layers are observed at the end of AUG campaigns.

The highest IR power fluxes are measured about $5 \mathrm{~cm}$ away from the inner strike point, where they exceed the probe measurements by a factor of 5 , see figure 8 . The modelled inner target power load is in between the IR and LP measurements, but the power fluxes are overestimated at the strike point. The discrepancy at the inner strike point is most distinct in comparison with the measured $\mathrm{D}_{\epsilon}$ emission, which increases steadily throughout the discharge and exceeds the modelled emission by a factor of 10. The emission is underestimated in the simulations even when $100 \%$ recycling and values of $n_{\text {sep }}$ up to $2.0 \times 10^{19} \mathrm{~m}^{-3}$ are tested, in the hope of increasing the recycling fluxes. This suggests that processes resulting in high excitation states of neutrals, such as volume recombination, might not be properly described in the simulations.

We summarise the results of the first benchmarking of reversed field divertor modelling for AUG. In reversed field, medium-recycling divertor conditions are measured at both targets. Qualitatively, these regimes can be reproduced in the modelling for both divertor targets simultaneously. Furthermore, it appears that a better match to the measured in-out asymmetries in target temperature and density can be achieved in reversed field compared to forward field with a similar, mediumrecycling regime in the outer divertor. However, significant discrepancies are observed when comparing the detailed target profiles obtained in the modelling against multiple diagnostic measurements. The discrepancies at the outer target cannot be removed if a simultaneous match to the measured upstream profiles of $n_{\mathrm{e}}$ and $T_{\mathrm{e}}$ is requested. These discrepancies question the validity of the solution particularly around the upper ${ }^{13} \mathrm{CH}_{4}$ injection location, where also the measured conditions are uncertain. Further experimental data in reversed field, covering a wider density regime, would be needed in order to assess these discrepancies in detail.

\section{Parallel ion flows and radial electric field}

The discrepancies observed at the targets might be associated with various deficiencies in the modelled divertor physics, and work is on-going to investigate these issues further. However, also strong parallel flows, measured in the SOL of various tokamaks [24-26], could play a role in, for example, transporting impurities from one target to another. Despite dedicated efforts in numerical interpretation (see e.g. $[27,28])$, the origin and effect of these flows on the divertor power and flux asymmetries is not yet fully understood. In the following, the observed SOL flows in AUG are compared to the SOLPS5.0 simulations.

Parallel Mach numbers were measured in the two field configurations by a reciprocating probe head, RP, positioned $0.3 \mathrm{~m}$ above the LFS torus midplane [29]. Figure 9 shows the measured Mach number, the plasma parameters $n_{\mathrm{e}}$ and $T_{\mathrm{e}}$ measured by additional, swept probe tips, and the $E_{r}$ profile deduced from the probe measurements of floating potential, assuming $V_{\mathrm{p}}=V_{\mathrm{f}}+3 T_{\mathrm{e}} / e$ [20]. The Mach number was derived according to the Hutchinson's formula [30]:

$$
M=\frac{v_{\|}}{c_{\mathrm{s}}}=0.43 \ln \frac{J_{\mathrm{sat}}^{+}}{J_{\text {sat }}^{-}},
$$

where the + and - signs refer to measurements made on the two opposite faces of 

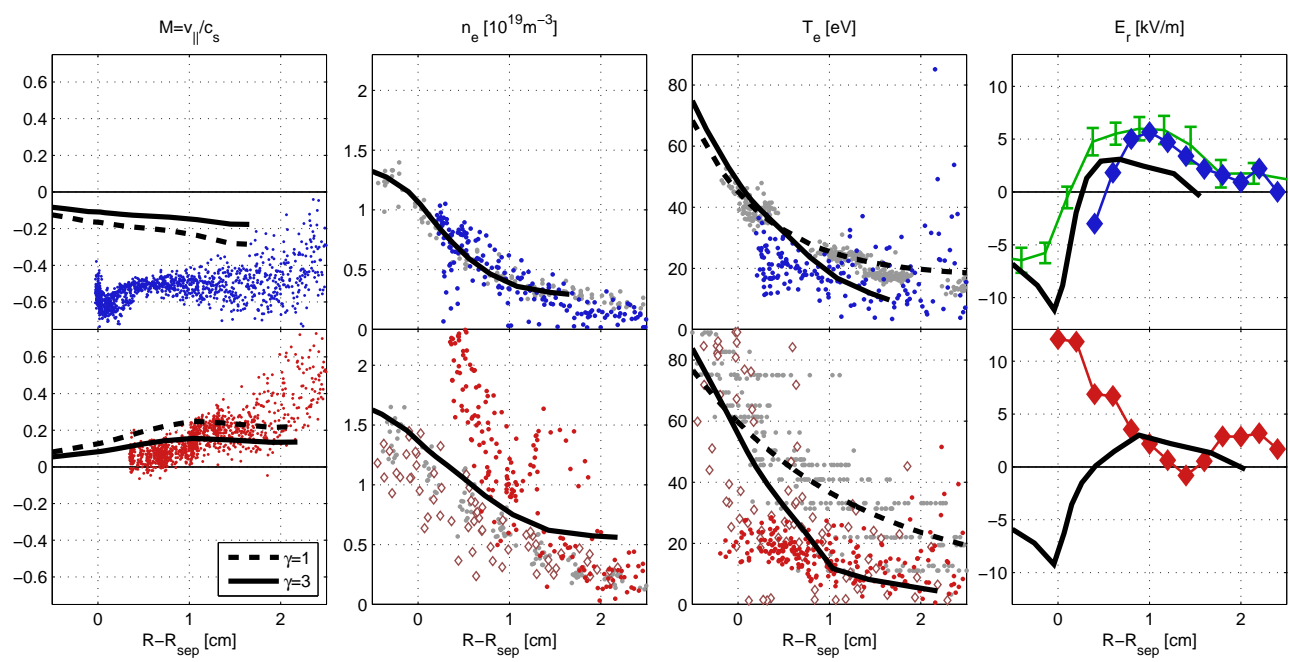

Figure 9. Measurements made by a probe head installed in the AUG midplane manipulator in forward (upper row: \#24908, blue points) and reversed field (lower row: \#25881, red points). From left to right: $M_{\|}$derived from Mach probes, $n_{\mathrm{e}}$ and $T_{\mathrm{e}}$ extracted from swept probes, and $E_{r}$ calculated from the probe measurements of $V_{\mathrm{f}}$. The green curve shows the $E_{r}$ profile from reflectometry in forward field. For comparison, the grey points show the $n_{\mathrm{e}}$ and $T_{\mathrm{e}}$ measurements by the Li-beam and ECE diagnostics, respectively, and the hollow diamonds represent the TS data (see table 1 for discharge numbers). The dashed solid lines show the fits to the $T_{\mathrm{e}}$ data measured by TS and ECE, which are used when evaluating the probe $E_{r}$. The simulation results are drawn in black, using two different models for $\gamma$ in the sound speed formula (2).

the probe head. The sign convention is chosen such that negative Mach numbers refer to flow towards the inner divertor. Only one swept probe was operational in these experiments, but the calculated density presents an average between the two faces, assuming that the temperature is the same on both sides of the probe and, hence, the measured density ratio between the faces is equal to the $J_{\text {sat }}$ ratio in (3). According to figure 9, the probe underestimates the $T_{\mathrm{e}}$ values close to the separatrix. In reversed field, also the density is overestimated compared to data from other diagnostics. These discrepancies appear to be due to difficulties in fitting the current-voltage characteristics in these plasma conditions, as noted also in other discharges [31]. Therefore, we have calculated the plasma potentials using $T_{\mathrm{e}}$ profiles fitted to the TS and ECE data. The fits are shown with the dashed lines. In forward field, also the $E_{r}$ derived from Doppler reflectometry [32], DR, is shown.

In forward field, strong parallel flows towards the inner divertor are measured, with $M \sim-0.5$ and a constant flow pattern from $0.5 \mathrm{~cm}$ to $3 \mathrm{~cm}$ outside the separatrix. In contrast, the measurements in reversed field give an almost stagnant plasma close to the separatrix, with an increase to $M \sim 0.2$ around $1 \mathrm{~cm}$ and further to $M \sim 0.5$ at $2.5 \mathrm{~cm}$, directed towards the outer divertor. The results are consistent with earlier reports from AUG, indicating an increase in the forward field flow velocity with decreasing $\bar{n}_{\mathrm{e}}$, and fall in between reports from other tokamaks, where in reversed field either large flows towards the outer target (TCV, below LFS midplane [25]) or smaller flows towards the inner target (JET, crown [26]) have been observed. The 
simulations agree qualitatively with the measured flow directions but, in contrast to the experiment, the modelled flows are symmetrically reversed with the field reversal. In forward field, the flow velocities are underestimated compared to the experiment by a factor of $2-5$. The quantitative agreement is, however, surprisingly good in reversed field.

Large discrepancies between modelled and measured forward field flows have been identified in several earlier publications (see e.g. [26,27]). In [27], the underestimation of $E_{r}$ by the fluid codes was pointed out as a likely cause for the underestimated flow velocities in forward field. According to figure 9, the modelled $E_{r}$ in forward field is $2-5 \mathrm{kV} / \mathrm{m}$ below the measured $E_{r}$ values in the SOL, the largest discrepancy being in the well depth inside the separatrix. The quantitative agreement is sensitive to the validity of the theoretical models involved in the experimental analysis, and to the accuracy of the fits used in the data analysis. For instance, kinetic effects or secondary electron emission could lead to deviations from the assumed values of the sheath potential $\left(V_{\mathrm{sh}} \sim-3 T_{\mathrm{e}} / e\right)$ [33]. Furthermore, temperature fluctuations, which have been measured close to the outer midplane separatrix in ASDEX Upgrade, could influence the measurements [34]. Both diagnostics and the code solution agree, however, with the shape of the $E_{r}$ profile, taking into account the radial uncertainty in the measurements, particularly regarding the probe $E_{r}$. As all other low-field-side measurements are well matched by the code, the main uncertainty is in the evaluation of the Mach number, either by the probes or by the code.

The improved match to the flows in reversed field compared to forward field has been suggested earlier when modelling Alcator C-mod [35] and JET [36]. In this paper it has been shown that, despite this good agreement, the match to the low-field-side divertor conditions is worse than in forward field with similar $\bar{n}_{\mathrm{e}}$. In reversed field, the probe measures a flat $V_{\mathrm{f}}$ profile at the innermost locations, leading to a strong positive electric field that contradicts the code profile. Again, we acknowledge the possibility that $E_{r}$ is not realistically described by the simplified formula used in the probe analysis, or the $T_{\mathrm{e}}$ gradient is overestimated, possibly combined with a radial shift of the data. However, also the discrepancies at the target could lead to an incorrectly modelled $E_{r}$ at the midplane. Based on these comparisons in the two field directions, we cannot identify a clear link between the $E_{r}$ and Mach number discrepancies. Furthermore, we cannot identify a link between the Mach number and divertor discrepancies. This stresses the importance of future investigations on flows and $E_{r}$ fields with field reversal.

Finally, we show in figure 10 the modelled electric field components perpendicular to the magnetic field and the parallel flow velocities at the outer target. These are discussed in particular to support the analysis of local impurity migration that will be presented in Part II. Accordingly, the electric field components are calculated in the directions parallel to the target surface, towards the outer SOL $\left(E_{x}\right)$, and normal to the target, towards the tiles $\left(E_{z}\right)$. Due to flux expansion, the perpendicular electric field components are significantly weaker at the target compared to the plasma midplane. However, it will be shown in Part II that these field components at the target are sufficiently large to produce significant $\mathbf{E} \times \mathbf{B}$ drifts of impurities. One sees that in forward field, the two electric field components are equally large, whereas in reversed field, the electric field is dominated by the component towards the surface. This close to the target, the component parallel to the surface is largely influenced by variations in the sheath potential along the surface. The absence of this component in reversed field can thus be attributed to the flat target temperature profile. Due to the lower 


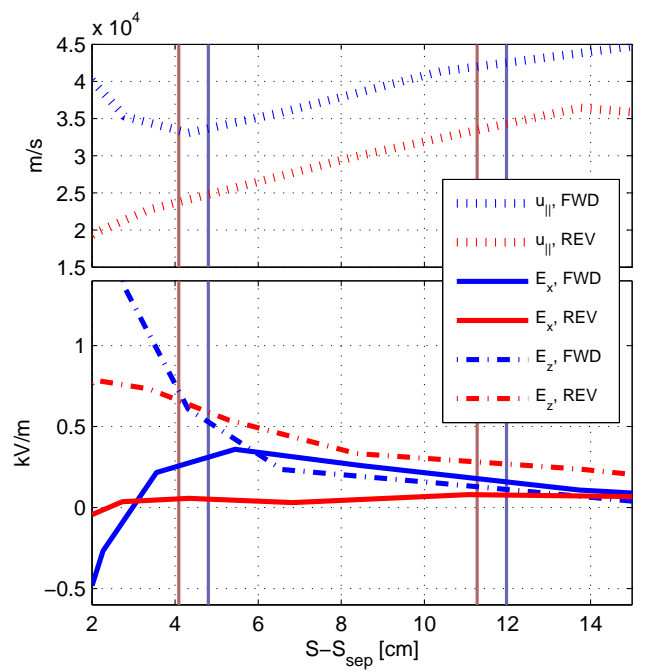

Figure 10. Modelled parallel flow velocities and electric field components along the outer divertor target in forward (blue) and reversed field (red). The ${ }^{13} \mathrm{CH}_{4}$ injection locations are drawn with the vertical blue and red lines, respectively. Note that here the $x$-direction is taken to be along the target surface and the $z$-direction is towards the surface. Due to flux expansion, $E_{r}$ is smaller at the target compared to upstream. The $E_{x}$ produces an $\mathbf{E} \times \mathbf{B}$ drift towards (forward field) or out of (reversed field) the surface. The $E_{z}$ produces an $\mathbf{E} \times \mathbf{B}$ drift along the surface [14].

target temperature, the parallel flow velocities are smaller in reversed field compared to forward field.

\section{Conclusions}

The paper presented a detailed comparison between SOLPS5.0 code calculations and low-density L-mode discharges in AUG. The reversal of the toroidal magnetic field and plasma current was found to significantly increase the density in the outer divertor plasma, for similar main plasma conditions. The SOLPS5.0 simulations focused in particular on reproducing the observations in the outer divertor in these two field directions, in order to realistically model the $2 \mathrm{D}$ distribution of plasma parameters for later analysis of local impurity migration (to be presented in Part II). Solutions that matched the multiple measurements in the outer divertor and simultaneously agreed with the upstream density and temperature measurements were sought for.

In forward field, a solution that agreed well with all measurements in the lowrecycling outer divertor could be achieved, when using assumptions and boundary conditions compatible with the measured upstream profiles of $n_{\mathrm{e}}$ and $T_{\mathrm{e}}$, within their uncertainty ranges. This implies a good consistency between the various diagnostic data in the low-recycling outer divertor. The density in the high-recycling inner divertor was simultaneously overestimated by a factor of 2 and, despite the good overall agreement in the low-field side of the plasma, the modelled parallel ion flows at the outer midplane were a factor of $2-5$ lower than in the experiment. Similar discrepancies between modelling and measurements have been observed in the past. However, it is assumed that they do not significantly compromise our model for the 
outer divertor, which will be used for the impurity migration studies presented in Part II.

In reversed field, both divertors were in a medium-recycling regime, and the measured divertor particle and power loads were reproduced in the simulations within a factor of 2 . This is a closer agreement with experimental data than what is typically observed for a similar outer divertor regime in forward field. However, discrepancies in the $2 \mathrm{D}$ distribution of divertor plasma parameters were identified at both targets. In particular, the simulations could not reproduce the observed accumulation of density in the outer SOL of the outer divertor, when a simultaneous match to upstream measurements was requested. The modelling agreed, however, with the measured parallel ion flows in the main SOL, which were found to be smaller and in the opposite direction compared to forward field.

From these studies, we conclude the following. A consistent set of outer divertor diagnostic measurements can be obtained in forward field, if the target is in low-recycling regime. A code solution matching these multiple outer divertor measurements can be achieved while simultaneously requesting a match to the measured upstream profiles of $n_{\mathrm{e}}$ and $T_{\mathrm{e}}-$ an observation which appears to be restricted to low-recycling divertor regimes. We note that the fits to the upstream profiles can typically be varied within a wider range in low-density L-mode plasmas compared to H-mode plasmas with steep pedestal, see [7]. However, even when taking significant liberties in assigning the upstream conditions, we could not achieve a similarly good match to the measurements in the medium-recycling outer divertor in reversed field. Furthermore, we could not identify a link between the discrepancies in outer divertor and those in the upstream Mach number, as the latter was well reproduced only in reversed field. Further studies of both forward and reversed field plasmas with flow measurements will be needed to address the missing physics ingredients in detail.

\section{Acknowledgments}

The authors would like to thank Dr. A. Chankin for useful discussions. This work, supported by the European Communities under the contracts of Association between EURATOM-Tekes and IPP-EURATOM, was carried out within the framework of the Task Forces on Plasma Wall Interactions and Integrated Tokamak Modelling of the European Fusion Development Agreement. The views and opinions expressed herein do not necessarily reflect those of the European Commission. The work was partially funded by the Academy of Finland project No. 134930.

\section{References}

[1] Schneider R et al, 2006 Contrib. Plasma Phys. 46(1-2) 3-191

[2] Taroni A et al, 1992 Contributions to Plasma Physics 32(3-4) 438-443

[3] Rognlien T D et al, 1994 Contributions to Plasma Physics 34(2-3) 362-367

[4] Kukushkin A et al, 2009 Nuclear Fusion 49(7) 075008

[5] Kirschner A et al, 2009 J. Nucl. Mater. 390-391 152-155

[6] Wischmeier M et al, $2009 \mathrm{~J}$. Nucl. Mater. 390-391 250 - 254

[7] Chankin A V et al, 2006 Plasma Phys. Control. Fus. 48 839-868

[8] Wischmeier M et al, 2011 Journal of Nuclear Materials 415(1, Supplement) S523 - S529

[9] Wiesen S et al, 2011 Journal of Nuclear Materials 415(1, Supplement) S535 - S539

[10] Herrmann A, 2003 Fusion Science and Technology Special Issue 44(3)

[11] Carthy P J M and ASDEX Upgrade Team Plasma Physics and Controlled Fusion 54(1) 015010 
[12] Marchand R and Dumberry M, 1996 Computer Physics Communications 96 232-246

[13] Aho-Mantila L et al, 2010 Contrib. Plasma Phys. 50(3-5) 439-444

[14] Aho-Mantila L et al, 2011 J. Nucl. Mater. 415(1, Supplement) S231 - S234

[15] Leuterer F et al, 2001 Fusion Engineering and Design 56-57 615 - 619

[16] Fischer R et al, 2008 Plasma Physics and Controlled Fusion 50(8) 085009

[17] Schneider P A et al, 2012 Differences in the h-mode pedestal width of temperature and density. Submitted to Plasma Phys. Control. Fus.

[18] Stangeby P C and Chankin A V, 1995 Physics of Plasmas 2(3) 707-715

[19] Weinlich M and Carlson A, 1997 Phys. Plasmas 4(6) 2151-2160

[20] Stangeby P, 2000 The Plasma Boundary of Magnetic Fusion Devices. IOP Publishing Ltd, Bristol

[21] Harhausen J, 2008 Interpretation of $D_{\alpha}$ Imaging Diagnostics Data on the ASDEX Upgrade Tokamak. Ph.D. thesis, Ludwig-Maximilians-Universität München

[22] Roth J, 1999 Journal of Nuclear Materials 266-269 51 - 57

[23] Kirschner A et al, 2011 J. Nucl. Mater. 415(1, Supplement) S239 - S245

[24] Asakura N, 2009 Plasma and Fusion Research 4 021-021

[25] Pitts R et al, 2007 Journal of Nuclear Materials 363-365 505 - 510

[26] Erents S K et al, 2004 Plasma Physics and Controlled Fusion 46(11) 1757

[27] Chankin A et al, 2007 Nuclear Fusion 47(8) 762

[28] Tamain P et al, 2009 Journal of Nuclear Materials 390-391 347 - 350

[29] Müller H W et al, 2007 Journal of Nuclear Materials 363-365 605 - 610

[30] Hutchinson I H, 1988 Phys. Rev. A 37(11) 4358-4366

[31] Müller H W et al, 2007 34th EPS Conf. on Plasma Physics P1.060

[32] Conway G D, Poli E and Happel T, 2010 Plasma and Fusion Research 5(S2005)

[33] LaBombard B et al, 2004 Nuclear Fusion 44(10) 1047

[34] Nold B et al, 2011 ArXiv e-prints, submitted to New Journal of Physics Influence of temperature fluctuations on plasma turbulence investigations with Langmuir probes

[35] Bonnin X et al, 2005 Journal of Nuclear Materials 337-339 301 - 304

[36] Bonnin X et al, 2005 In ECA, ed., Proceedings of the 32nd EPS Conference on Plasma Phys. Tarragona, vol. 29C. ECA. P-2.110 